\title{
Aspect-Based Sentiment Analysis Using Smart Company and Hotel Aspect Review Data
}

\author{
C. Selvi, ${ }^{,}$, Niveda. C. $\mathrm{P}^{2}$
}

${ }^{*}$ Department of CSE, Velalar College of Engineering and Technology, Erode, Tamil Nadu, India ${ }^{2}$ PG Scholar, Department of CSE, Velalar College of Engineering and Technology, Erode, Tamil Nadu, India

\begin{abstract}
Digital sources such as smart applications opinions and online feedback statistics are crucial resources to be seeking for customers' remarks and input. However, the reviews are often disorganized, leading to difficulties in information navigation and knowledge acquisition. The aforementioned problem is overcome by generating aspect-sentiment based embedding for the hotels and companies by looking into reliable reviews of them. The important product aspects are identified based on two observations: 1) the important aspects are usually commented on by a large number of consumers and 2) consumer opinions on the important aspects greatly influence their overall opinions. Aspect frequency and the influence of consumer opinions given to each aspect over their overall opinions are identified for hotel reviews whereas for company reviews approach adopts language processing techniques, policies, and lexicons to address several sentiment evaluation challenges, and convey summarized results. Moreover, aspect ranking achieve significant performance improvements, which demonstrate the capacity of aspect ranking in facilitating real-world applications.
\end{abstract}

Keywords : Aspect frequency, aspect ranking, language processing technique, aspect-sentiment based embedding.

\section{INTRODUCTION}

\section{A.DATA MINING}

Data mining is a process of searching large data to discover patterns for simple analysis. Data mining is a technology to help companies focus on their data warehouse. So it is called as Knowledge Discovery in Data (KDD). KDD decisions are allowed by data mining tools for businesses. Data mining tools can answer business questions that traditionally were time consuming to resolve.

\section{B.OPINION MINING}

Opinion mining is a process of tracking the mood of the public about a particular product. Opinions can be essential when it's use to make a decision or choose among multiple option. Information-gathering behavior has always been to find out what other people think. The availability of opinion-rich resources such as online review sites and personal blogs, and challenges arise, to understand the opinions of others people.

\section{C.OPINION MINING AND ANALYSIS}

Mining the sentiment information in the massive user generated content can help to sense the public's opinions towards a mixture of topics, such as products, brands, disasters, events and so on. 
Classifying the sentiments of massive micro blog messages is also helpful to substitute or supplement traditional polling, which is expensive and timeconsuming. These methods have been used to analyze the sentiments of product reviews, micro blogs and so on. The global sentiment classifier is shared by all domains and is trained on the labeled samples from various domains to have better generalization ability.

\section{D.EVALUATIONS OF SENTIMENT CLASSIFICATION ON PRODUCT ASPECTS}

User compared the following methods of sentiment classification: (a) one unsupervised method (b) three supervised methods Multi Model Naïve Bayes (MMNB)(K-SVM), Maximum Entropy (ME), and Support Vector Machine (SVM). In particular, SVM was implemented by using SVM with linear kernel, K-SVM was implemented with Laplace smoothing, and ME was implemented with L-BFGS parameter estimation. User can see that the three supervised methods perform much better than the unsupervised approach.

\section{E.COMPANY ASPECT OPINION MINING}

Sentiment task is about predicting the polarity of a piece of text usually positive or negative. People have studied sentiment prediction at the document level, sentence level and phrase level. This is an extremely popular task in the field of company aspect Opinion Analysis. The goal is to provide a summary in the form of star ratings or scores on each of these features. So the task involves finding features and then discovering the sentiments for each feature. The comments or reviews are helpful or insightful. Instead of displaying these comments or user reviews in chronological order, sorting the reviews by its helpfulness would improve user productivity. The goal of the task is to automatically predict the helpfulness of user reviews instead of just relying on user votes. Company aspect Opinion based-entity ranking is the task of ranking entities based on opinions. The query is essentially "preferences" for the entity. The results would be the likelihood of the entities matching those preferences. So opinions on the entities match the specified preferences, the higher the rank.

\section{RELATED WORKS}

\section{A. A LIBRARY FOR SUPPORT VECTOR MACHINES}

LIBSVM is a library for Support Vector Machines (SVMs). User has been actively developing this package since the year 2000. The goal is to help users to easily apply SVM to their applications. LIBSVM has gained wide popularity in machine learning and many other areas. In this article, user presents all implementation details of LIBSVM. Issues such as solving SVM optimization problems, theoretical convergence, multi-class classification, probability estimates, and parameter selection are discussed in detail classic IR (Information Retrieval) is inherently predicated on users searching for information, the socalled "information need". But the need behind a user search is often not informational -- it might be navigational (give me the url of the site I want to reach) or transactional (show me sites where I can perform a certain transaction, e.g. shop, download a file, or find a map). Users explore this taxonomy of user searches and discuss how global search engines evolved to deal with user-specific needs.

\section{B. MULTI-DOCUMENT SERVICE USAGE CLASSIFICATION OF EVALUATIVE TEXT}

Present and compare two approaches to the task of summarizing evaluative arguments. The first is a sentence extraction based approach while the second is a language generation-based approach. User 
evaluates these approaches in a user study and finds that they quantitatively perform equally user. Qualitatively, however, user finds that they perform user different but complementary reasons. User concludes that an effective method for summarizing evaluative arguments must effectively synthesize the two approaches. Document polarity classification poses a significant challenge to data-driven methods, resisting traditional text-categorization techniques (Pang, Lee, and Vaithyanathan, 2002). Previous approaches focused on selecting indicative lexical features (e.g., the word "good"), classifying a document according to the number of such features that occur anywhere within it. In contrast, user propose the following process: (1) label the sentences in the document as either subjective or objective, discarding the latter; and then (2) apply a standard machine-learning.

\section{THE ANATOMY OF A LARGE-SCALE HYPERTEXTUAL USER SEARCH ENGINE}

Google is designed to crawl and index the user efficiently and produce much more satisfying search results than existing systems. The prototype with a full text and hyperlink database of at least 24 million pages is available at http: llgoogle.stanford.edu/ To engineer a search engine is a challenging task. Search engines index tens to hundreds of millions of user pages involving a comparable number of distinct terms. They use tens of millions of queries every day. Despite the importance of large-scale search engines on the user, very little academic research has been done on them. Furthermore, due to rapid advance in technology and user proliferation, creating a user search engine today is very different from three years ago. This work provides an in-depth description of our large-scale user search engine - the first such detailed public description user know of to date.

\section{D.SUPERVISED AND UNSUPERVISED ASPECT CATEGORY DETECTION FOR SENTIMENT ANALYSIS WITH CO- OCCURRENCE DATA}

Using online consumer reviews as electronic word of mouth to assist purchase-decision making has become increasingly popular. The Web provides an extensive source of consumer reviews, but one can hardly read all reviews to obtain a fair evaluation of a product or service. A text processing framework that can summarize reviews would therefore be desirable. A subtask to be performed by such a framework would be to find the general aspect categories addressed in review sentences, for which this paper presents two methods. In contrast to most existing approaches, the first method presented is an unsupervised method that applies association rule mining on co-occurrence frequency data obtained from a corpus to find these aspect categories. While not on par with state-of-the-art supervised methods, the proposed unsupervised method performs better than several simple baselines, a similar but supervised method, and a supervised baseline, with an F 1 -score of $67 \%$. The second method is a supervised variant that outperforms existing methods with an F 1 -score of 84\%.

\section{E. ASPECT TERM EXTRACTION FOR SENTIMENT ANALYSIS IN LARGE MOVIE REVIEWS}

With the rapid development of the World Wide Web, electronic word-of-mouth interaction has made consumers active participants. Nowadays, a large number of reviews posted by the consumers on the Web provide valuable information to other consumers. Such information is highly essential for decision making and hence popular among the internet users. This information is very valuable not only for prospective consumers to make decisions but also for businesses in predicting the success and sustainability. In this paper, a Gini Index based feature selection method with Support Vector Machine (ELM) classifier is proposed for sentiment 
classification for large movie review data set. The results show that our Gini Index method has better classification performance in terms of reduced error rate and accuracy. However, to effectively handle the large amount of information available in these reviews, a framework for the automated summarization of reviews is desirable.

\section{F. ASPECT-BASED SENTIMENT ANALYSIS TO REVIEW PRODUCTS USING NAIVE BAYES}

Product reviews can provide great benefits for consumers and producers. Number of reviews could be ranging from hundreds to thousands and containing various opinions. These make the process of analyzing and extracting information on existing reviews become increasingly difficult. In this research, sentiment analysis was used to analyze and extract sentiment polarity on product reviews based on a specific aspect of the product. This research was conducted in three phases, such as data preprocessing which involves part-of-speech (POS) tagging, feature selection using Chi Square, and classification of sentiment polarity of aspects using Naïve Bayes. Based on evaluation results, it is known that the system is able to perform aspect-based sentiment analysis with its highest F1-Measure of 78.12\%.

\section{PROPOSED SYSTEM}

In proposed work for hotels, a reviews aspect ranking framework is made to automatically identify the important reviews of reviews from online consumer reviews.

The Multi Model Naïve Bayes based opinion review analysis reviews possess the following characteristics: (b) Users opinions on these reviews greatly influence their overall opinions on the reviews. A straightforward frequency-based solution is to regard the reviews that are frequently commented in consumer reviews as important.

For company opinion mining, an integrated lexicon and rule-based aspect-based ELM sentiment analysis approach is used to extract company aspect and classify the corresponding implicit and explicit sentiments. This approach is selected due to the nature of the targeted dataset, which consists of short reviews and irregular sentences related to the various aspects of the company aspect mobile apps. This project highlighted the significance of the rule-based over other approaches as it depends on manually.

Otherwise, if the opinion word cannot determine the aspect category, the algorithm will search for the nearest aspect term in the same sentence with maximum window size of two with more priority to the right side, since the adjective usually occurs before the term.

The pair of identified opinion word and aspect term will be looked up in the lexicon in order to determine the aspect category illustrates the algorithm to extract the explicit and implicit aspects. This function returns two arrays where the first array (aspect Indices) represents the indices of the aspect terms in the review and the second array (aspect Categories) represents the as aspect categories to the corresponding aspect terms in the first array will provide better result.

(a) They are frequently commented in consumer reviews and 


\section{MODULE DESCRIPTION}

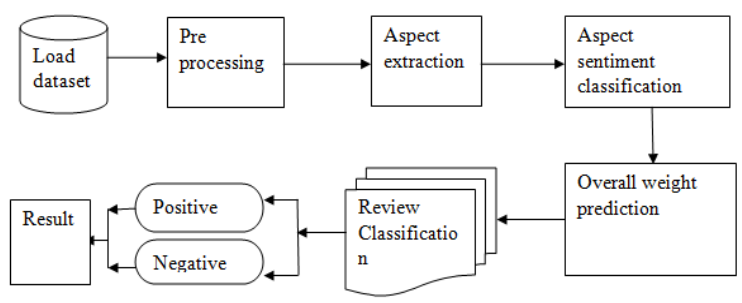

\section{System Architecture}

\section{A.PREPROCESSING DATASET AND ASPECT IDENTIFICATION}

Naive Bayes product aspect ranking extractive summarizers aim at picking out the most relevant sentences in the reviews while also maintaining a low redundancy in the summary. In this module it summarizers aim at picking out the most relevant sentences in the reviews while also maintaining a low redundancy in the summary. Finally, stop words will be marked to be out of any of the following phases by using a customized list of stop words such as "the", "an" and "of". This list has been initiated by studying the domain and the reviews in the dataset. In this module to generate generic summary, nonstop-words that occur most frequently in the reviews(s) may be taken as the query words. Since these words represent the theme of the reviews, they generate generic summaries. Term frequency is usually 0 or 1 for sentences-since normally the same content-word does not appear many times in a given sentence. If users create query words the way they create for information retrieval, then the query based summary generation would become generic summarization. In this module aspect identification (identifying specific words and phrases that refer to aspects of the review subject) and aspect-based sentiment analysis (determining the sentiment of each aspect).

\section{B.NAIVE BAYES PRODUCT ASPECT RANKING APPROACH:}

Reviews are usually written such that they address different topics one after the other in an organized manner. They are normally broken up explicitly or implicitly into sections. This organization applies even to summaries of reviews. It is intuitive to think that summaries should address different "themes" appearing in the reviews. Some summarizers incorporate this aspect through classification. If the review collection for which summary is being produced is of totally different topics, reviews classification becomes almost essential to generate a meaningful summary. Reviews are represented using Term Frequency Inverse Reviews Frequency (TF-IDF) of scores of words. Term frequency used in this context is the average.

\section{C.ELM BASED IMPLICIT AND EXPLICIT ASPECT EXTRACTION}

Aspects categories are vital for the aspect extraction task in sentiment analysis. To address this requirement in the company aspect mobile apps domain have defined a set of aspects categories according to the written standards by Android, Apple and Smart Dubai Office. The resulting aspects categories were User Interface, User Experience, Functionality and Performance, Security, and Support and Updates. These aspects categories are used in this study.

\section{D.ASPECT SENTIMENT SCORING}

The approach that has been followed employs the populated lexicons reduced by . Basically, the algorithm as Function 2represents, navigates through the sentences and once an opinion word is identified, its polarity score is retrieved through the lexicon and linked with the extracted aspect. In the experiment, we applied several settings to the algorithm in order to identify opinion words in a sentence in addition to the use of the lexicons. For instance, various rules are 
adopted to handle negations, intensification, down toners, repeated characters, and the special case of negation-opinion rules. The usage of ELM has multiple benefits like comparable or better performance than other machine learning models like SVMs, and most importantly boasts a significant reduction in model building time. Training time is an important aspect in our work given its high probability to be adapted into an online and real-time application.

\section{E.REVIEW CLASSIFICATION:}

It helps to classify every reviews. VWNB algorithm treats texts as Bags Of Words (BOW). Where the order of words and as such context is ignored. The original text is filtered down to only the words that are thought to carry sentiment. Each review can be categorical/Polarity - was that bit of text "positive", "neutral" or "negative?" In this process, you are trying to label a piece of text as either positive or negative or neutral. In this module A set of consumer reviews are composed in different formats on various forum Websites. The Websites such as CNet.com require consumers to give an overall rating on the product, describe concise positive and negative opinions (i.e. Pros and Cons) on some product aspects, as well as write a paragraph of detailed review in free text.

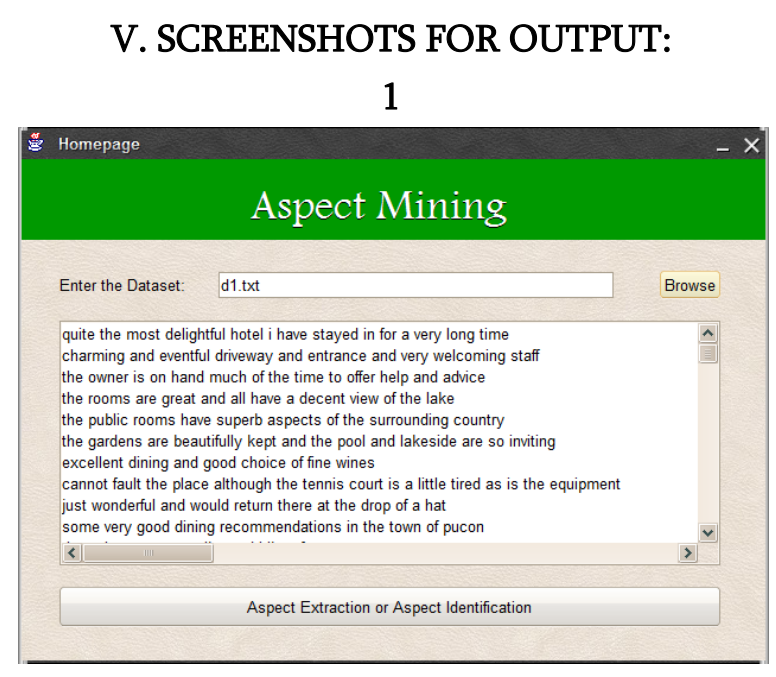

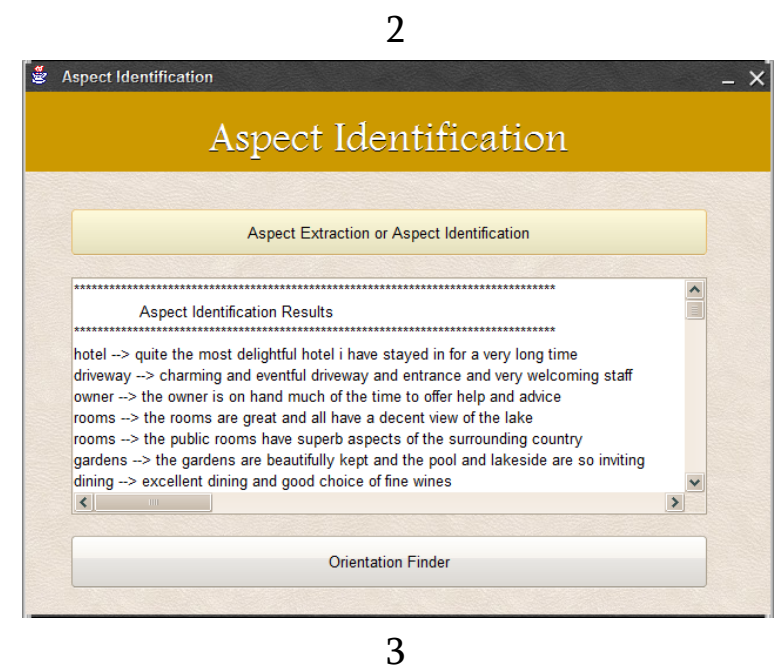

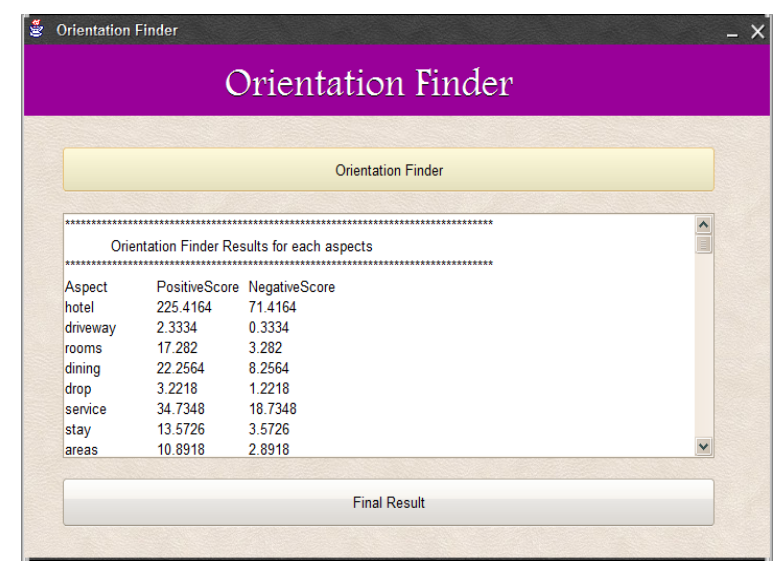

4

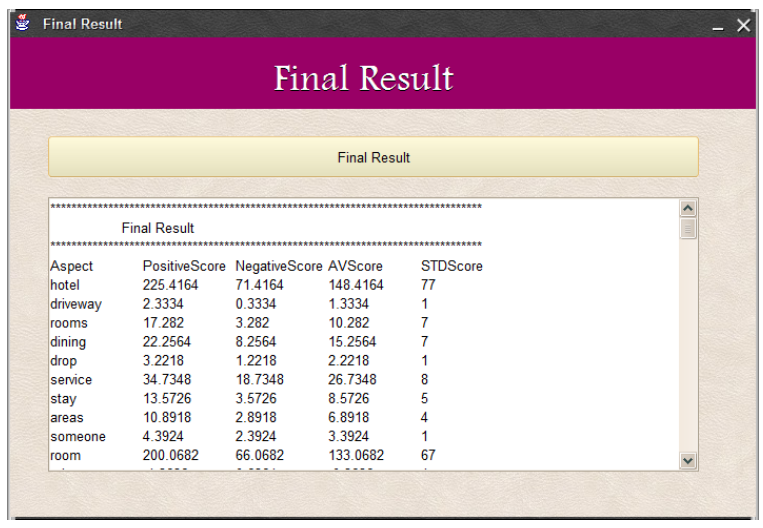

\section{CONCLUSION}

Aspect-primarily based sentiment analysis is taken into consideration as one of the difficult tasks in sentiment analysis area of research. It is crucial that each one feedback are understood and categorized so that smart company aspects can rely on this channel 
to concentrate to their customers. Therefore, this can be taken into consideration as a aspect for future smart services improvements and optimizations that exceed the people's expectations. In this regard, an integrated lexicon and rule-based method turned into hired to extract explicit and implicit issue as well as sentiment type for these elements. The dataset contains a extensive number of similar sentences. This can be taken into consideration as additional rules that can expand the proposed model. One brief and easy manner is to activate the users for presenting a review within the cell app.

\section{REFERENCES}

[1]. D. M. Blei, L. Carin and D. Dunson (2010), "Probabilistic topic models", IEEE Signal Process. Mag., vol. 27, no. 6, pp. 55-65, 2010.

[2]. D. Borth, R. Ji, T. Chen, T. Breuel, S.-F. Chang, "Large-scale visual sentiment ontology and detectors using adjective noun pairs", Proc. 21st ACM Int. Conf. Multimedia, pp. 223-232, 2013.

[3]. Z. Chen, A. Mukherjee, B. Liu, M. Hsu, M. Castellanos, R. Ghosh, "Leveraging multidomain prior knowledge in topic models", Proc. 23th Int. Joint Conf. Artif. Intel., pp. 2071-2077, 2011.

[4]. P. Isola, J. Xiao, A. Torralba, A. Oliva, "What makes an image memorable?", Proc. IEEE Conf. Comput. Vis. Pattern Recognit., pp. 145-152, 2013.

[5]. Y. Jia, M. Salzmann, T. Darrell, "Learning crossmodality similarity for multinomial data", Proc. 13th IEEE Int. Conf. Comput. Vis., pp. 24072414, 2014.

[6]. A.S. Manek, P.D. Shenoy, M.C. Mohan, K.R. Venugopal, Aspect term extraction for sentiment analysis in large movie reviews using Gini Index feature selection method and ELM classifier, World Wide Web. 20 (2017)135-154, https://doi.org/10.1007/s11280-015-0381-x.
[7]. M.S. Mubarok, M. Adiwijiaya, AldhiDwi, Aspect-based sentiment analysis to review products using Naïve bayes, in: AIP Conference Proceedings, AIP Publishing, 2017, pp. 1-8, https://doi.org/10.1063/1.4994463.

[8]. D. Pham, A. Le, Data \& knowledge engineering learning multiple layers of knowledge representation for aspect based sentiment analysis, DataKnowl. Eng. 114 (2018)26-39, https://doi.org/10.1016/j.datak.2017.06.001.

[9]. M. Rathan, V.R. Hulipalled, K.R. Venugopal, L.M. Patnaik, Consumer insight mining: aspect based Twitter opinion mining of mobile phone reviews, Appl. Soft Comput. J. 68 (2018) 765773, https://doi.org/10.1016/j. asoc.2017.07.056.

[10]. K. Schouten, O. van der Weijde, F. Frasincar, R. Dekker, Supervised and unsupervised aspect category detection for sentiment analysis with co- occurrence data, IEEE Trans. Cybern. 48 (2018) 1263-1275, https://doi.org/ 10.1109/TCYB.2017.2688801.

\section{Cite this article as :}

C. Selvi, Niveda. C. P, "Aspect-Based Sentiment Analysis Using Smart Company and Hotel Aspect Review Data", International Journal of Scientific Research in Science and Technology (IJSRST), Online ISSN : 2395-602X, Print ISSN : 2395-6011, Volume 7 Issue 2, pp. 112-118, March-April 2020. Available at doi : https://doi.org/10.32628/IJSRST207223 Journal URL : http://ijsrst.com/IJSRST207223 